\title{
Prevalence and factors associated with nonalcoholic fatty liver disease in shanghai work-units
}

\author{
Xiaona Hu ${ }^{1,2+}$, Yiqin Huang ${ }^{1,2+}$, Zhijun Bao ${ }^{1,2^{*}}$, Yiqian Wang ${ }^{1,2}$, Dongmei Shi ${ }^{1,2}$, Fang Liu ${ }^{1,2}$, Zhanjuan Gao ${ }^{1,2}$ \\ and Xiaofeng $Y^{1,2}$
}

\begin{abstract}
Backgrounds: Nonalcoholic fatty liver disease (NAFLD) has become the most common chronic liver disease in Asians. However, data on prevalence and factors associated with NAFLD in Asians are lacking. The aim of this study is to investigate the prevalence of NAFLD in Shanghai employees to assess the relationship between NAFLD and age, gender, metabolic risk factors in this studied population.

Methods: We selected 7152 employees of Shanghai work-units. Each of them underwent detailed medical history-taking, physical examination, laboratory assessments and abdominal ultrasonography. The diagnosis of NAFLD was done according to established criteria. Receiver operating characteristics (ROC) curves were applied to detect areas under the ROC curves for each index. Nominal logistic regression analysis was used to estimate the odds ratio for risk factors of NAFLD.

Results: About $38.17 \%$ employees had NAFLD, more in men than in women. The prevalence of NAFLD increased with increasing age. In both genders, the prevalence of metabolic factors was higher in the NAFLD group. Body max index, waist circumference, weight-to-height ratio, blood pressure, blood glucose, total cholesterol, triglyceride, low density lipoprotein, high density lipoprotein and uric acid were found to have a diagnostic value for NAFLD. Body max index is a better index for diagnosing NAFLD. Uric acid is a new diagnosing index not inferior to lipid metabolic factors. Metabolic factors can increase the risk of NAFLD up to $1.5 \sim 3.8$ times.
\end{abstract}

Conclusions: Older age, male gender, metabolic factors such as obesity, abdominal obesity, dyslipidemia, hypertension or type 2 diabetes are risk factors for NAFLD. Prevalence of NAFLD in Shanghai employees is high. Prevention is extremely important. Those achieve the critical point should have early intervention.

Keywords: Prevalence, Risk factors, Nonalcoholic fatty liver

\section{Backgrounds}

Nonalcoholic fatty liver disease (NAFLD) is a metabolic disorder characterized by excessive triglyceride accumulation in hepatocytes. Nowadays it has become a major public health hazard worldwide. Estimates of current prevalence rates range from $24 \%$ to $42 \%$ in Western countries and 5\% to $40 \%$ in Asian countries [1-3]. In the

\footnotetext{
* Correspondence: xinyi8681@sina.com

${ }^{\dagger}$ Equal contributors

'Department of Gastroenterology, Huadong Hospital, Fudan University, Shanghai, 200040, China

${ }^{2}$ Department of Internal Medicine, Shanghai Medical College, Fudan

University, 221 West Yan An Road, Jing'an District, Shanghai 200040, China
}

USA, it is estimated that approximately a third of the general population has NAFLD [2,4]. In Japan, the prevalence is $9 \sim 30 \%[1,5,6]$. And the prevalence of NAFLD in the general population of China varies from $5 \%$ to $24 \%$. A combination of lifestyle, environmental, older age, gender, steroid hormone metabolism, genetic predisposition and metabolic factors play a role in the pathogenesis of NAFLD [7-10]. Genetic predisposition, overabundance of calorie-rich food and lack of physical activity contribute to development of obesity. Obesity is a pro-inflammatory state that leads to insulin resistance (IR), which is closely associated with NAFLD development and progression [7,11]. Advancing age decreases 
the hepatic metabolism of LDL cholesterol, increases abdominal adiposity and fat deposition in muscles, liver, and bone marrow, resulting in deleterious metabolic consequences of IR [8]. An ethnic variation in the distribution of NAFLD has also been suggested with Hispanics having the highest prevalence (45-58\%), followed by Caucasians (33-44\%) and African Americans (24-35\%) $[12,13]$.

NAFLD consists of a wide spectrum of conditions, ranging from simple steatosis to nonalcoholic steatohepatitis (NASH) which can progress to cirrhosis and hepatocellular carcinoma (HCC). It is reported that almost $10 \% \sim 20 \%$ of individuals with NAFLD have NASH, $10 \% \sim 15 \%$ of individuals with NASH progress to cirrhosis [14]. In patients with cirrhotic NASH, HCC and liver failure are the main causes of morbidity and mortality (5-year cumulative HCC development rate 11.3\%, 5-year survival rate $75.2 \%$, respectively) [15]. A "two-hit hypothesis" was proposed to explain the pathogenesis and progression of NAFLD. It was proposed that hepatocyte triglyceride accumulation resulting from metabolic imbalance (obesity, IR and diabetes) is the leading factor to steatosis (the "first hit") and that the lipid-laden hepatocytes are then vulnerable to injurious processes (the "second hit") such as cytokines and oxidative stress [7]. In this hypothesis, obesity and insulin resistance are the key pathogenic factors associated with NAFLD.

NAFLD has a multifactorial etiology and a combination of environmental, genetic and metabolic factors play a role in the development of advanced disease. So the pathophysiology of NAFLD is not fully understood. Therefore, study of the prevalence of NAFLD and identifying its risk factors would be critically important. China is a country with a large scale and many nations. Regional variations can be striking. Shanghai is an international city with more westernized lifestyle. The prevalence may be higher than the general population. Therefore, we conducted a study to determine the prevalence of and factors associated with NAFLD in Shanghai employees.

\section{Methods}

\section{Subject recruitment}

We assigned a number to each of 16 urban districts of Shanghai, and randomly selected three districts (Xuhui Districts, Jing'an Districts and Changning Districts). Of all the work-units we randomly selected three departments in each district. Then we performed medical check-ups at Huadong Hospital during 2011.

About 7152 adult participants, aged 18 65 years old, were enrolled in the study. The study was approved by the Research Ethics Committee of Huadong Health Bureau. The purpose of the study, procedures to be carried out, potential hazards and benefits were explained prior to obtaining an informed written consent. Each consenting adult underwent a detailed medical history-taking, physical examination, laboratory assessment and abdominal ultrasonography (US) carried out by hepatologists trained at the same institution to ensure interobserver consistency.

Information was gathered on sociodemographic variables, past history of liver disease, presence of comorbidities, medical history and health-related habits such as smoking and drinking. Age, sex, occupation and education were gathered as sociodemographic variables. Past history covered previous or present diagnoses of hepatitis $\mathrm{B}$ or $\mathrm{C}$ infection, biliary diseases, surgical interventions and other chronic liver diseases. Presence of co-morbidities included hypertension, type 2 diabetes (T2DM) and obesity. Alcohol intake was assessed using two open questions: 'How often did you have a drink containing alcohol per week in the past 6 months?' and 'How many glasses did you have on a typical day when you were drinking in the past 6 months?' In the second question, one glass of alcoholic beverage was assumed to contain $10 \mathrm{~g}$ of alcohol. From these two questions, we calculated an average daily intake of alcohol.

In accordance with the guidelines, Subjects diagnosed with NAFLD had to fulfill the following criteria: no history of current or past excessive alcohol consumption, defined by an average daily consumption of alcohol intake $>20 \mathrm{~g} /$ day $(140 \mathrm{~g} /$ week $)$ in males and $>10 \mathrm{~g} /$ day (70g/week) in females; no history of systemic illness known to cause fatty liver disease; not receiving or have recently received hepatotoxic drugs; negative tests for the presence of hepatitis B surface antigen and antibody to hepatitis $\mathrm{C}$ virus; absence of history and clinical, biochemical and US findings consistent with cirrhosis and other chronic(autoimmune, celiac disease, genetic disorders such as Wilson's disease and alpha-1-antitrypsin deficiency) liver diseases; fulfilled the criteria with fatty liver under abdominal ultrasonography.

\section{Physical examination}

Weight, standing height and waist circumference (WC) were measured in a standardized fashion by a trained examiner. WC measurement was made midway between the last rib and the iliac crest. The standing height and WC measurement were made at minimal inspiration to the nearest $0.1 \mathrm{~cm}$. Body mass index (BMI) was calculated as weight $(\mathrm{kg}) /$ stature $\left(\mathrm{m}^{2}\right)$, and weight-to-Height ratio calculated as WC divided height. Readings of clinical blood pressure (BP) were obtained in the left arm of patients in the supine position, after 5 min of quiet rest, with a mercury sphygmomanometer. A minimum of three BP readings were taken on three separate occasions at least 2 weeks apart. Baseline BP values were the 
average of the last two of three consecutive measurements obtained at intervals of $3 \mathrm{~min}$.

\section{Laboratory assessments}

Antecubital venous blood samples were taken from all subjects after a 12h overnight fast. Using a multichannel autoanalyzer, we measured serum levels of alanine transarninase (ALT), total cholesterol (TC), triglyceride (TG), Low Density Lipoprotein cholesterol (LDL-C), High Density Lipoprotein cholesterol (HDL-C), fasting plasma glucose (FPG), creatinine (Cr) and uric acid (UA). Those participants with evidence of hepatic steatosis or abnormal blood tests of liver function had further investigations performed including serology of hepatitis B and C, ceruloplasmin, ferritin, alpha-1-antritrypsin level and phenotype and autoimmune markers such as antinuclear antibody (ANA), antismooth muscle antibody (SMA), and antimitochondrial antibody (AMA). Oral glucose tolerance test (OGTT) was performed on the subjects with abnormal fasting glucose except those with a previous diagnosis of diabetes.

\section{Ultrasonographic examination}

Abdominal US was performed in all subjects by two hepatologists who were trained at the same institution and unaware of the clinical and laboratory data. Fatty liver was diagnosed in the presence of two of the three following criteria: increased hepatic echogenicity compared to the spleen or the kidneys, blurring of liver vasculature and deep attenuation of the ultrasonographic signal [16]. When the hepatic steatosis reaches 33\%, the detection sensitivity is nearly $100 \%$. So this has an adequate threshold for detection of steatosis when more than $33 \%$ of hepatocytes contain fat on liver histology $[17,18]$.

\section{Definitions}

According to current guidelines, patients with a systolic blood pressure $(\mathrm{SBP}) \geq 140 \mathrm{mmHg}$ and/or diastolic blood pressure $(\mathrm{DBP}) \geq 90 \mathrm{mmHg}$ were defined as hypertensive [19]. Causes of secondary hypertension were excluded by clinical and biochemical tests. Impaired fasting glucose and T2DM were defined by the American Diabetes Association criteria revised in 2003 [20]. Metabolic abnormalities were diagnosed following the Third Report of the National Cholesterol Education Expert Panel on Detection, Evaluation, and Treatment of High Blood Cholesterol in Adults, modified by the criteria of obesity proposed for Asians by the Steering Committee of the Regional Office for the Western Pacific Region of WHO (WPRO) [21,22]. The diagnosis of NAFLD was based on guidelines for the assessment and management of nonalcoholic fatty liver disease in the Asia-Pacific region $[23,24]$.

\section{Statistical analyses}

All statistical analyses were performed using SPSS 16.0. Data is expressed as the mean \pm standard deviation or median [inter-quartile range (25\%-75\%)] or as percentage. Differences between groups were tested using an independent two-sample t-test or Maan-Whitney U-test for continuous variables, and the Pearson chi-square test was used to test for differences in the distribution of categorical variables. Nonparametric methods were carried out for non-normally distributed values. In each gender, receiver operating characteristics (ROC) curves were applied to detect the sensitivity, specificity and areas under the ROC curves (AUCs) for each index (normal vs abnormal) and NAFLD (absent vs present). Nominal logistic regression analysis was applied to analyze independent relationships of risk factors for NAFLD. All provided $P$-values represent the results of two-sided tests. $P$-values $<0.05$ were considered statistically significant.

\section{Results}

General characteristics of participants

Overall, 7152 subjects enrolled in the study, 4330 subjects were men, while 2822 subjects were women. As shown in Table 1, the prevalence of NAFLD in general was $38.17 \%$ (47.88\% for men and $23.28 \%$ for women). There is a significant difference in gender between NAFLD and non NAFLD group $(51.04 \%$ for male and $48.96 \%$ for female in non NAFLD group,75.93\% for male and $24.07 \%$ for female in NAFLD group, $\chi^{2}=437.87$, $\mathrm{p}<0.0001$ ). In males, the average age of the subjects with NAFLD was $46.82 \pm 13.50$, and $40.29 \pm 14.76$ in those without NAFLD. In females, the average age in NAFLD and non NAFLD group was $50.95 \pm 11.58$ and $38.28 \pm 12.15$ respectively. There were statistical differences in BMI, WC, weight-to-height ratio, SBP, DBP, TG, TC, LDL, HDL, UA, FPG and ALT between NAFLD group and non NAFLD group in both genders. There was no difference in Cr. Metabolic factors such as BMI, BP, TG, TC and FPG in NAFLD group were significantly higher than non NAFLD group.

\section{Age-specific prevalence of NAFLD}

As showed in Figure 1, the prevalence of NAFLD increased according to age (trend chi-square value $=$ 23.7292, $\mathrm{p}<0.0001$ in total; chi-square value $=15.4859$, $\mathrm{p}<0.0001$ in male; trend chi-square value $=19.0515$, $\mathrm{p}<0.0001$ in female). The peak prevalence was in the $50-65$ age group, up to $54 \%$ of subjects had NAFLD in total. The prevalence of NAFLD in males was significantly higher than females within the same age group $(p<0.0001)$. 
Table 1 Baseline characteristics, biochemical tests and metabolic characteristics of subjects

\begin{tabular}{|c|c|c|c|c|c|c|}
\hline & \multicolumn{3}{|c|}{ Male $(n=4330)$} & \multicolumn{3}{|c|}{ Female $(n=2822)$} \\
\hline & Non NFALD(n=2257) & NALFD $(n=2073)$ & $P$-value & Non NALFD( $n=2165)$ & NALFS $(n=657)$ & $P$-value \\
\hline Age (years) & $40.29 \pm 14.76$ & $46.82 \pm 13.50$ & $<0.01$ & $38.28 \pm 12.15$ & $50.95 \pm 11.58$ & $<0.01$ \\
\hline BMI $\left(\mathrm{kg} / \mathrm{m}^{2}\right)$ & $22.56 \pm 2.81$ & $26.12 \pm 2.88$ & $<0.01$ & $21.24 \pm 2.56$ & $25.34 \pm 2.88$ & $<0.01$ \\
\hline WC $(\mathrm{cm})$ & $78.69 \pm 9.79$ & $89.69 \pm 11.45$ & $<0.01$ & $70.79 \pm 6.85$ & $81.14 \pm 9.79$ & $<0.01$ \\
\hline W/H & $0.46 \pm 0.06$ & $0.52 \pm 0.06$ & $<0.01$ & $0.44 \pm 0.04$ & $0.51 \pm 0.06$ & $<0.01$ \\
\hline $\mathrm{SBP}(\mathrm{mmHg})$ & $117.42 \pm 14.64$ & $127.99 \pm 17.08$ & $<0.01$ & $113.05 \pm 14.49$ & $128.44 \pm 17.70$ & $<0.01$ \\
\hline $\mathrm{DBP}(\mathrm{mmHg})$ & $71.99 \pm 12.03$ & $80.45 \pm 12.43$ & $<0.01$ & $69.09 \pm 10.48$ & $76.87 \pm 11.27$ & $<0.01$ \\
\hline $\mathrm{TG}(\mathrm{mmol} / \mathrm{L})$ & $1.0[0.8 \sim 1.5]$ & $1.8[1.3 \sim 2.6]$ & $<0.01$ & $0.8[0.6 \sim 1.1]$ & $1.5[1.1 \sim 2.1]$ & $<0.01$ \\
\hline $\mathrm{TC}(\mathrm{mmol} / \mathrm{L})$ & $4.40 \pm 0.81$ & $4.89 \pm 0.89$ & $<0.01$ & $4.51 \pm 0.84$ & $5019 \pm 1.00$ & $<0.01$ \\
\hline $\mathrm{ALT}(\mathrm{U} / \mathrm{L})$ & $19[14 \sim 25]$ & $29[21 \sim 44]$ & $<0.01$ & $13[10 \sim 17]$ & $21[16 \sim 30]$ & $<0.01$ \\
\hline $\mathrm{UA}(\mu \mathrm{mol} / \mathrm{L})$ & $353.00 \pm 69.93$ & $385.16 \pm 76.64$ & $<0.01$ & $261.27 \pm 52.11$ & $303.21 \pm 67.56$ & $<0.01$ \\
\hline$\overline{\mathrm{FPG}}(\mathrm{mmol} / \mathrm{L})$ & $4.94 \pm 0.93$ & $5.35 \pm 1.39$ & $<0.01$ & $4.83 \pm 0.71$ & $5.37 \pm 1.34$ & $<0.01$ \\
\hline $\mathrm{CR}(\mu \mathrm{mol} / \mathrm{L})$ & $75.86 \pm 10.76$ & $75.36 \pm 11.54$ & 0.146 & $55.01 \pm 7.84$ & $55.21 \pm 8.82$ & 0.586 \\
\hline $\mathrm{HDL}(\mathrm{mmol} / \mathrm{L})$ & $1.78 \pm 0.42$ & $1.43 \pm 0.36$ & $<0.01$ & $1.98 \pm 0.40$ & $1.57 \pm 0.38$ & $<0.01$ \\
\hline $\mathrm{LDL}(\mathrm{mmol} / \mathrm{L})$ & $2.73 \pm 0.69$ & $3.13 \pm 0.77$ & $<0.01$ & $2.80 \pm 0.71$ & $3.31 \pm 0.85$ & $<0.01$ \\
\hline
\end{tabular}

Values, mean \pm standard error of the mean (S.E.M).

BMI: body mass index; WC: waist circumference; W/H: weight to height ratio; SBP: systolic blood pressure; DBP: diastolic blood pressure; TG: Triglycerides; TC: total cholesterol; UA: Uric acid; FPG: Fasting plasma glucose; CR: Creatinine; HDL: High Density Lipoprotein cholesterol; LDL: Low Density Lipoprotein cholesterol.

\section{Prevalence of metabolic syndrome}

As shown in Table 2, the prevalence of metabolic factors in non NAFLD population was $8.19 \%$ for obesity, $7.61 \%$ for abdominal obesity, $11.18 \%$ for $\mathrm{BP}>130$ / $85 \mathrm{mmHg}$ or hypertension, $8.74 \%$ for dyslipidemia and $3.71 \%$ for $\mathrm{FPG}>5.60 \mathrm{mmol} / \mathrm{L}$ or T2DM. The prevalence of NAFLD combined with metabolic factors was $22.44 \%$ for obesity, $17.93 \%$ for abdominal obesity, $18.64 \%$ for $\mathrm{BP}>130 / 85 \mathrm{mmHg}$ or hypertension, $19.85 \%$ for dyslipidemia and $7.69 \%$ for $\mathrm{FPG}>5.60 \mathrm{mmol} / \mathrm{L}$ or T2DM respectively.

In males, the prevalence of metabolic factors in the NAFLD group was significantly higher than in non NAFLD group (obesity $29.53 \%$ vs $9.68 \%$, abdominal obesity $22.31 \%$ vs $7.81 \%$, BP > $130 / 85 \mathrm{mmHg}$ or hypertension $23.37 \%$ vs $11.50 \%$, dyslipidemia $26.26 \%$ vs $9.15 \%$ and $\mathrm{FBG}>5.60 \mathrm{mmol} / \mathrm{L}$ or T2DM $9.70 \%$ vs $4.20 \%$ respectively). In females, the comparison of the prevalence of metabolic factors in the NAFLD group and non NAFLD group was $11.55 \%$ vs $5.92 \%$ for obesity, $11.20 \%$ vs $7.30 \%$ for abdominal obesity, $11.37 \%$ vs $10.70 \%$ for $\mathrm{BP}>130 / 85 \mathrm{mmHg}$ or hypertension, $10.03 \%$ vs $8.11 \%$ for dyslipidemia and $4.61 \%$ vs $2.94 \%$ for $\mathrm{FBG}>5.60 \mathrm{mmol} / \mathrm{L}$ or T2DM.

The difference of the prevalence of metabolic factors in the males and females NAFLD group was $29.53 \%$ vs $11.55 \%$ for obesity, $22.31 \%$ vs $11.20 \%$ for abdominal obesity, $23.37 \%$ vs $11.37 \%$ for $\mathrm{BP}>130 / 85 \mathrm{mmHg}$ or hypertension, $26.26 \%$ vs $10.03 \%$ for dyslipidemia and $9.70 \%$ vs $4.61 \%$ for $\mathrm{FBG}>5.60 \mathrm{mmol} / \mathrm{L}$ or $\mathrm{T} 2 \mathrm{DM}$.
Receiver operating curve analyses of age, biochemical tests and metabolic characteristics

Table 3 shows the AUC values of the subjects with NAFLD. After analysis, we found that the AUC values for BMI was higher than other metabolic factors in both genders $(p<0.05)$. In males, there were no significant statistical differences in weight-to-height ratio and WC $(\mathrm{u}=1.51, \mathrm{p}=0.066)$. The AUC values for TG, TC, HDL, LDL were higher than UA $(p<0.01)$. There were no significant statistical differences in FPG and UA. In females, there were also no significant statistical differences in weight-to-height ratio and WC $(\mathrm{u}=1.33$, $\mathrm{p}=0.093$ ). The AUC values for TG and HDL were higher than UA $(p<0.01)$, but there were no significant statistical differences in TC, LDL, FPG and DBP.

\section{The adjusted odds ratios of metabolic risk factors for NAFLD}

Table 4 shows the risk factors for NAFLD after being adjusted for age. In males, NAFLD was more likely to occur in subjects with high TG levels (OR 3.809), those with obesity (OR 3.304), abdominal obesity (OR 1.960), high blood pressure (OR 1.949), high FPG (OR 1.502) and low HDL (OR 1.596). Hypertriglyceridemia is the strongest associated factor in males, while BMI is the strongest associated factor in females (OR 3.806), followed by Hypertriglyceridemia (OR 3.381), abdominal obesity (OR 2.846), high FPG (OR 2.458), high BP (OR 2.053), and low HDL (1.801). 


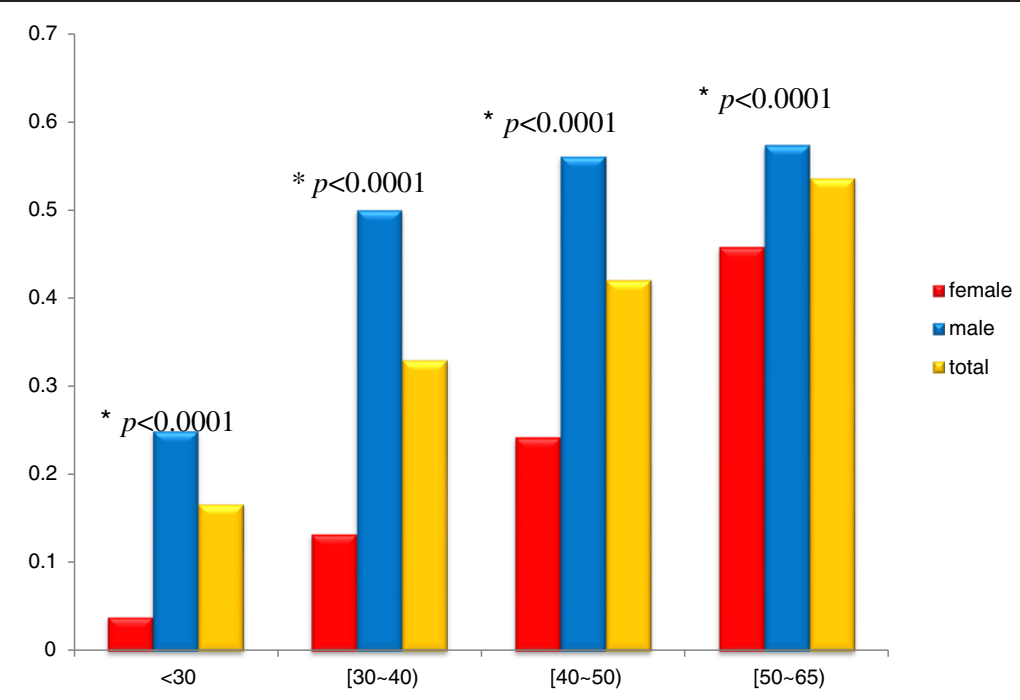

Figure 1 Age-specific prevalence of NAFLD. The prevalence of NAFLD increased according to age(trend chi-square value $=23.7292$, $p<0.0001$ in total; chi-square value $=15.4859, p<0.0001$ in male; trend chi-square value $=19.0515, p<0.0001$ in female). The peak prevalence was in the 50-65 age group, up to 54\% persons had NAFLD in total. The prevalence of NAFLD in males was significantly higher than females within the same age group (25\% vs 3.89\% in younger than 30- year old age group, 50\% vs $13.29 \%$ in $30-39$ age group, $56.06 \%$ vs $24.27 \%$ in $40-49$ age group, and $57.35 \%$ vs $45.79 \%$ in $50-64$ age group). In female, the prevalence increased dramatically after 50 years old. *The contraction between male and female in the same age group, $p<0.0001$.

\section{Discussion}

Several studies have shown that NAFLD is the main cause of chronic liver disease [25]. In China, the prevalence of NAFLD varied widely due to differences in occupation, age, gender, life-style and regions studied [26]. Previous studies had reported the prevalence of NAFLD in the general population of central China, Chengdu (Southwest China), Guangdong (South China) and Shanghai (East China) as $24.5 \%, 12.5 \%, 17 \%$ and $15 \%$ respectively [27-29]. Regional variations within China can be striking. Due to the difficulty of carrying out large scale population survey, the true prevalence of the general population in China is still absent. In this study, we investigated the prevalence of NAFLD in employees in Shanghai to evaluate the trends of NAFLD prevalence rates. Our study reveals that the present prevalence of NAFLD in Shanghai is $38.17 \%$, much higher than the previous study. Our study also investigated the prevalence of the components of metabolic syndrome. The prevalence of obesity, hypertension, dyslipidemia and T2DM are 30.63\%, 29.82\%, 28.59\% and $11.4 \%$ respectively. Therefore, the difference of the present and previous prevalence could be attributed to the increasing prevalence of obesity, hypertension, insulin resistance and T2DM.

Our study investigated the employees in an age range of 18-64 years old. The study confirmed that the prevalence of NAFLD increases with increasing age and the peak prevalence of NAFLD was between 50-65 years old. However, it is difficult to generalize our results to the very old as our subjects were mainly the 'younger old' with few being aged $>65$ years. Further studies focusing specifically on the 'old' with NAFLD are needed.

Table 2 Prevalence of metabolic syndrome

\begin{tabular}{|c|c|c|c|c|c|c|c|c|c|}
\hline & \multicolumn{3}{|c|}{ Male } & \multicolumn{3}{|c|}{ Female } & \multicolumn{3}{|c|}{ Total } \\
\hline & $\begin{array}{l}\text { NAFLD } \\
(\mathrm{n}(\%))\end{array}$ & $\begin{array}{c}\text { Non NAFLD } \\
(\mathrm{n}(\%))\end{array}$ & $\mathbf{P}$ & $\begin{array}{l}\text { NAFLD } \\
(\mathrm{n}(\%))\end{array}$ & $\begin{array}{c}\text { Non NAFLD } \\
(\mathbf{n}(\%))\end{array}$ & $\mathbf{P}$ & $\begin{array}{l}\text { NAFLD } \\
(\mathrm{n}(\%))\end{array}$ & $\begin{array}{c}\text { Non NAFLD } \\
(\mathrm{n}(\%))\end{array}$ & $P$ \\
\hline obesity & 1279(29.53) & $419(9.68)$ & .000 & $326(11.55)$ & $167(5.92)$ & .000 & $1605(22.44)$ & $586(8.19)$ & .000 \\
\hline Abdominal obesity & $966(22.31)$ & 338(7.81) & .000 & $316(11.20)$ & 206(7.30) & .000 & $1282(17.93)$ & $544(7.61)$ & .000 \\
\hline $\mathrm{BP}>130 / 85$ or $\mathrm{HBP}$ & $1012(23.37)$ & 498(11.50) & .000 & $321(11.37)$ & $302(10.70)$ & .000 & 1333(18.64) & $800(11.18)$ & .000 \\
\hline dyslipidemia & $1137(26.26)$ & 396(9.15) & .000 & 283(10.03) & $229(8.11)$ & .000 & 1420(19.85) & $625(8.74)$ & .000 \\
\hline $\mathrm{FPG}>5.6$ or $2-\mathrm{DM}$ & $420(9.70)$ & $182(4.20)$ & .000 & $130(4.61)$ & 83(2.94) & .000 & $550(7.69)$ & 265(371) & .000 \\
\hline
\end{tabular}

Metobolic syndrome: (1) Obesty: Body mass index $\geq 25 \mathrm{~kg} / \mathrm{m} 2$; abdominal obesity: $\geq 90 \mathrm{~cm}($ male), $\geq 80 \mathrm{~cm}(\mathrm{female})$; (2) Blood pressure (BP) $\geq 130 / 85 \mathrm{mmHg}$ Or hypertension (HBP); (3) Dyslipidemia: Triglycerides (TG) $\geq 1.7 \mathrm{mmol} / \mathrm{L}$, and/or High Density Lipoprotein cholesterol (HDL) $\leq 1.03 \mathrm{mmol} / \mathrm{L}$, (male) $<1.29 \mathrm{mmol} / \mathrm{L}$ (female); (4) Fasting blood glucose (FPG) $\geq 5.6 \mathrm{mmol} / \mathrm{L}$ Or type 2 diabetes (2-DM). 
Table 3 The AUC values of metabolic risk factors for NAFLD

\begin{tabular}{|c|c|c|c|c|c|c|c|c|}
\hline \multirow[b]{2}{*}{ Variable(s) } & \multicolumn{4}{|c|}{ Male } & \multicolumn{4}{|c|}{ Female } \\
\hline & Area $^{\dagger}$ & S.E $^{\ddagger}$ & $95 \% \mathrm{Cl}^{\S}$ & $P$-value & Area $^{\dagger}$ & S.E $^{\ddagger}$ & $95 \% \mathrm{Cl}^{\S}$ & $P$-value \\
\hline BMI & .818 & .006 & $0.806 \sim 0.831$ & .000 & .867 & .007 & $0.853 \sim 0.882$ & .000 \\
\hline WC & .776 & .007 & $0.762 \sim 0.790$ & .000 & .830 & .009 & $0.813 \sim 0.847$ & .000 \\
\hline WC/Height & .791 & .007 & $0.778 \sim 0.804$ & .000 & .846 & .008 & $0.829 \sim 0.862$ & .000 \\
\hline SBP & .688 & .008 & $0.673 \sim 0.704$ & .000 & .757 & .011 & $0.736 \sim 0.778$ & .000 \\
\hline DBP & .690 & .008 & $0.674 \sim 0.706$ & .000 & .699 & .012 & $0.676 \sim 0.722$ & .000 \\
\hline$C R$ & .477 & .009 & $0.460 \sim 0.494$ & .008 & .503 & .013 & $0.477 \sim 0.529$ & .820 \\
\hline UA & .628 & .008 & $0.611 \sim 0.644$ & .000 & .688 & .012 & $0.665 \sim 0.711$ & .000 \\
\hline$\overline{F P G}$ & .628 & .008 & $0.612 \sim 0.645$ & .000 & .712 & .012 & $0.689 \sim 0.735$ & .000 \\
\hline $\mathrm{TG}$ & .775 & .007 & $0.761 \sim 0.789$ & .000 & .808 & .010 & $0.789 \sim 0.827$ & .000 \\
\hline TC & .665 & .008 & $0.649 \sim 0.681$ & .000 & .700 & .011 & $0.677 \sim 0.722$ & .000 \\
\hline $\mathrm{HDL}$ & .735 & .008 & $0.720 \sim 0.749$ & .000 & .772 & .010 & $0.752 \sim 0.792$ & .000 \\
\hline$\overline{\mathrm{LDL}}$ & .656 & .008 & $0.640 \sim 0.672$ & .000 & .687 & .012 & $0.663 \sim 0.710$ & .000 \\
\hline $\mathrm{TG} / \mathrm{HDL}$ & .777 & .007 & $0.763 \sim 0.791$ & .000 & .810 & .010 & $0.791 \sim 0.829$ & .000 \\
\hline
\end{tabular}

†The area under the ROC curve; $\$$ Standard error; $\S 95 \%$ confidence interval.

BMI: body max index; WC: waist circumference; WC /Height: weight to height ratio; SBP: systolic blood pressure; DBP: diastolic blood pressure; CR: Creatinine; UA: Uric acid; FPG: Fasting blood glucose; TG: Triglycerides; TC: total cholesterol HDL: High Density Lipoprotein cholesterol; LDL: Low Density Lipoprotein cholesterol.

Gender difference in the relationship between metabolic risk factors and NAFLD was another important finding in this study. A study conducted in a Korean population reported that the prevalence of NAFLD was $35 \%$ for men and $16 \%$ for women [30]. Our study revealed that the prevalence of NAFLD was estimated to be $47.88 \%$ for men and $23.28 \%$ for women, higher than Koreans. The data of higher prevalence of NAFLD among men compared to women was also supported from studies in USA, Japan and India [31-33]. This gender difference of prevalence maybe attributed to higher prevalence of metabolic syndrome in men [31]. The national difference in prevalence may be owing to ethnicity and lifestyle differences. In this study, the average age in the male NAFLD group was younger than the female group. In the same age group, the prevalence of NAFLD in men was higher than women. Logistic regression analysis of risk factors for NAFLD revealed that high TG level was the most relevant factor for NAFLD in men, while obesity was the most strongly associated factor for NAFLD in women (obesity defined by BMI $\geq 25 \mathrm{~kg} / \mathrm{m}^{2}$ ). These age and gender differences may be due to differences in prevalence of obesity and life-style-related disease.

All these differences above can be explained by genetic predisposition. A genetic underpinning for NAFLD is suggested by a number of studies [10]. In 2008, Romeo et al. conducted the first genome wide association study and reported the strongest genetic signal for the presence of fatty liver (PNPLA3, patatin-like phospholipase domain containing 3 ; rs738409). It was reported that PNPLA3 and four additional genetic variants had a modest role for lipid metabolism. The PNPLA3 gene is responsible for the difference in prevalence of fatty liver disease between ethnic groups. It could also be responsible for a lower prevalence of steatosis in males [34]. Apart from the PNPLA3 gene, many other genes can also influence the development of obesity and NAFLD

Table 4 The adjusted odds ratios of metabolic risk factors for NAFLD

\begin{tabular}{|c|c|c|c|c|c|c|c|c|}
\hline \multirow[b]{2}{*}{ Variable(s) } & \multicolumn{4}{|c|}{ Male } & \multicolumn{4}{|c|}{ Female } \\
\hline & $\beta$ & S.E & O.R & $P$-value & $\beta$ & S.E & O.R & $P$-value \\
\hline $\mathrm{BMI}$ & 1.195 & .014 & 3.304 & 0.000 & 1.336 & .023 & 3.806 & .000 \\
\hline WC & .673 & .014 & 1.960 & .000 & 1.046 & .022 & 2.846 & .000 \\
\hline $\mathrm{BP}$ & .667 & .011 & 1.949 & .000 & 0.719 & .017 & 2.053 & .000 \\
\hline FPG & .407 & .015 & 1.502 & .000 & 0.899 & .024 & 2.458 & .000 \\
\hline $\mathrm{TG}$ & 1.337 & .012 & 3.809 & .000 & 1.218 & .020 & 3.381 & .000 \\
\hline $\mathrm{HDL}$ & .468 & .021 & 1.596 & .000 & 0.588 & .024 & 1.801 & .000 \\
\hline normal & -6.239 & .036 & 0.002 & .000 & -8.096 & .048 & 0.000 & .000 \\
\hline
\end{tabular}

$\beta$ : Beta coefficient; S.E: Standard error; OR: odds ratio; BMl: Body mass index, $\geq 25 \mathrm{~kg} / \mathrm{m2} ;$ WC: waist circumference, $\geq 90 \mathrm{~cm}$ (male), $\geq 80 \mathrm{~cm}$ (female); BP: blood pressure, $\geq 130 / 85 \mathrm{mmHg}$ Or hypertension (HBP); FPG: Fasting blood glucose, $\geq 5.6 \mathrm{mmol} / \mathrm{L}$ Or type 2 diabetes (2-DM); TG: Triglycerides, $\geq 1.7 \mathrm{mmol} / \mathrm{L}$; HDL: High Density Lipoprotein, $\leq 1.03 \mathrm{mmol} / \mathrm{L}$, (male) $<1.29 \mathrm{mmol} / \mathrm{L}$ (female). 
via affecting lipid metabolism, cytokines, fibrosis mediators and oxidative stress $[10,35,36]$. Genetic factors shed a light in the identification of individuals at risk to develop NAFLD and its progression.

Many studies have proposed that the risk factors for NAFLD included a high fat diet, a sedentary lifestyle, insulin resistance, metabolic syndrome and its components (obesity, hypertension, dyslipidemia and T2DM) $[2,26,37]$. In our study, the ROC curves revealed that BMI, WC, weight-to-height ratio, BP, FPG, TC, TG, LDL, HDL and UA have diagnostic value for NAFLD. In western countries, visceral obesity had been shown to have a more important role in the pathogenensis of NAFLD than overall obesity. So WC has been a wellknown surrogate marker of abdominal fat accumulation. But our studies revealed that BMI was superior to WC, and BMI is a better index for diagnosing NAFLD. Recently, many studies recommended that weight-toheight was an index not affected by height, it may be a good index for detecting NAFLD. But our study found that there was no significant statistical difference in the AUCs of weight-to-height ratio and $\mathrm{WC}$, suggesting that waist-to-height ratio and $\mathrm{WC}$ has the same value for detecting NAFLD.

Recent studies have reported that serum uric acid levels were associated with NAFLD [38-41].The most significant finding of this study is that UA has a more diagnostic value for NAFLD. Our study found that there is no significant statistical difference in the AUCs of FPG and UA in males. UA is not inferior to FPG in diagnosing NAFLD. There are no significant statistical differences in the AUCs of UA and TC, LDL, BG, DBP in females. UA is not inferior to TC, LDL, BG and DBP in diagnosing NAFLD.

Our logistic regression analysis revealed that each component of metabolic syndrome was independently associated with NAFLD. Obesity, central obesity, BP $>130$ / $85 \mathrm{mmHg}$ and/or hypertension, dyslipidemia, high uric acid and FPG $>5.6 \mathrm{mmol} / \mathrm{L}$ and/or T2DM can increase the risk of NAFLD by $1.5 \sim 3.8$ times. Previous studies had reported that obesity was the strongest associated factor after adjusting for age, gender and other metabolic factors $[30,42,43]$. But we found that obesity was the most relevant factor in females, which can increase the risk of NAFLD by 3.8 times, while it is hypertriglyceridemia in males, which can increase the risk of NAFLD by 3.8 times. In males, obesity is secondary to hypertriglyceridemia, with 3.3 times risk increase for NAFLD.

The present study does have some limitations. First, we tried our best to exclude subjects with a history of habitual alcohol consumption in this study. However, since the limit of excessive alcohol use probably needs to be adjusted according to the size of body and gender, the exact amount of alcohol consumed and its alcoholic content were difficult to assess. Second, although histopathological findings remain the gold standard for diagnosing NAFLD, they cannot be applied to a large-scale population. Abdominal US is non-invasive, easily undertaken and has a sensitivity ranging from $60 \%$ to $94 \%$ and a specificity from $84 \%$ to $95 \%$ in detecting the presence of fatty liver. If the hepatic steatosis reaches $33 \%$, sensitivity approaches $100 \%$ [18]. So we used abdominal US to diagnose fatty liver. But when the infiltration is of less than $30 \%$ of the hepatic content, it is difficult to detect the presence of fatty liver. Finally, other confounders such as physical activity should also be considered. Further studies are required to adjust for these possibly distorted associations and to validate these findings to other populations.

\section{Conclusion}

In conclusion, NAFLD is the main etiology of chronic liver disease. The prevalence of NAFLD is increasing these years. The prevalence in Shanghai is much higher than the previous study in 2005. Older age, male gender, metabolic factors such as obesity, abdominal obesity, dyslipidemia, hypertension or type 2diabetes can increase the risk of NAFLD with $1.5 \sim 3.8$ times. The endstage of NASH is cirrhosis, HCC, and/or liver failure. NAFLD is also a risk factor for death caused by cardiovascular disease. Future work needs to deepen our understanding of the pathogenesis and progression, especially genetic determinants of NAFLD for prevention. Improved methods for early detection of NAFLD, such as improving imaging techniques, finding novel plasma biomarkers are urgently needed. The medicines for the treatment of NAFLD should continue to be tested to determine their long-term safety and efficacy and new optimal management of NAFLD is also important.

\section{Abbreviations \\ NAFLD: Nonalcoholic fatty liver disease; NASH: Nonalcoholic steatohepatitis; HCC: hepatocellular carcinoma; BMI: Body mass index; WC: waist circumference; BP: Blood pressure; SBP: systolic blood pressure; DBP: diastolic blood pressure; TG: Triglyceride; TC: Total cholesterol; HDL-C: High Density Lipoprotein cholesterol; UA: Uric acid; Cr: Creatinine; FPG: Fasting plasma glucose; ALT: Alanine transarninase; AST: Aspartate aminotransferase; ANA: antinuclear antibody; AMA: antismooth muscle antibody; OGTT: Oral glucose tolerance test; US: ultrasonographic scan; T2DM: type 2 diabetes; ROC: Receiver operating characteristics; AUCs: areas under the ROC curves.}

\section{Competing interests}

The authors declare that they have no competing interests.

\section{Authors' contributions}

Xiaona Hu undertook data collection, analyzed the interviews and wrote the manuscript and revised it upon the editor's and reviewers' guidance.Yiqin Huang collected data, transcribed and analyzed the interviews. Yiqian Wang and Dongmei Shi contributed to the study design and gave comments on the manuscript. Fang Liu, Zhanjuan Gao and Xiaofeng Yu undertook data collection and took part in the qualitative analysis of the interviews. Zhijun Bao conceived the initial idea, contributed to each stage of the study development, analysis, reporting, reviewed the analysis and interpretation of data, corrected the first draft, co-designed the contents and revised the 
manuscript. All authors have participated in the design of the study and have commented critically on the initial manuscript and have approved the final version of the manuscript.

\section{Funding}

Natural Science Foundation of Shanghai (11ZR1411600).

\section{Acknowledgments}

We acknowledge the Natural Science Foundation of Shanghai of their fund and the Health Bureau of Shanghai of their support. We also acknowledge the sonologists of Huadong Hospital and all the members of the Health Management Center of Huadong Hospital. We thank Jiaofeng Wang, Ph.D., for her assistance with statistical analysis. We thank Sheilesh K Dewan and Mohamed Kohla, for their critical revision of the manuscript.

Received: 8 February 2012 Accepted: 31 August 2012

Published: 14 September 2012

\section{References}

1. Amarapurkar DN, Hashimoto E, Lesmana LA, Sollano JD, Chen PJ, Goh KL: How common is non-alcoholic fatty liver disease in the Asia-Pacific region and are there local differences? J Gastroenterol Hepatol 2007, 22(6):788-793.

2. Fan JG, Saibara T, Chitturi S, Kim Bl, Sung JJ, Chutaputti A: What are the risk factors and settings for non-alcoholic fatty liver disease in Asia-Pacific? J Gastroenterol Hepatol 2007, 22(6):794-800.

3. Caballeria L, Auladell MA, Toran P, Miranda D, Aznar J, Pera G, Gil D, Munoz L, Planas J, Canut S, et al: Prevalence and factors associated with the presence of non alcoholic fatty liver disease in an apparently healthy adult population in primary care units. BMC Gastroenterol 2007, 7:41.

4. Bellentani S, Scaglioni F, Marino M, Bedogni G: Epidemiology of nonalcoholic fatty liver disease. Dig Dis 2010, 28(1):155-161.

5. Kojima S, Watanabe N, Numata M, Ogawa T, Matsuzaki S: Increase in the prevalence of fatty liver in Japan over the past 12 years: analysis of clinical background. J Gastroenterol 2003, 38(10):954-961.

6. Hamaguchi M, Kojima T, Takeda N, Nakagawa T, Taniguchi H, Fujii K, Omatsu T, Nakajima T, Sarui H, Shimazaki M, et al: The metabolic syndrome as a predictor of nonalcoholic fatty liver disease. Ann Intern Med 2005, 143(10):722-728

7. Guturu P, Duchini A: Etiopathogenesis of Nonalcoholic Steatohepatitis: Role of Obesity. Insulin Resistance and Mechanisms of Hepatotoxicity. Int J Hepatol 2012, 2012:212865.

8. Gan L, Chitturi S, Farrell GC: Mechanisms and implications of age-related changes in the liver: nonalcoholic Fatty liver disease in the elderly. Curr Gerontol Geriatr Res 2011, 2011:831536.

9. Hamaguchi M, Kojima T, Ohbora A, Takeda N, Fukui M, Kato T: Aging is a risk factor of nonalcoholic fatty liver disease in premenopausal women. World J Gastroenterol 2012, 18(3):237-243.

10. Hernaez R: Genetic factors associated with the presence and progression of nonalcoholic fatty liver disease: a narrative review. Gastroenterol Hepatol 2012, 35(1):32-41.

11. Nguyen TA, Sanyal AJ: Pathophysiology guided treatment of nonalcoholic steatohepatitis. J Gastroenterol Hepatol 2012, 27(Suppl 2):58-64

12. Williams CD, Stengel J, Asike MI, Torres DM, Shaw J, Contreras M, Landt CL, Harrison SA: Prevalence of nonalcoholic fatty liver disease and nonalcoholic steatohepatitis among a largely middle-aged population utilizing ultrasound and liver biopsy: a prospective study. Gastroenterology 2011, 140(1):124-131.

13. Browning JD, Szczepaniak LS, Dobbins R, Nuremberg P, Horton JD, Cohen JC, Grundy SM, Hobbs HH: Prevalence of hepatic steatosis in an urban population in the United States: impact of ethnicity. Hepatology 2004, 40(6):1387-1395

14. Pasumarthy L, Srour J: Nonalcoholic steatohepatitis: a review of the literature and updates in management. South Med J 2010 103(6):547-550.

15. Hashimoto $E$, Tokushige $K$ : Prevalence, gender, ethnic variations, and prognosis of NASH. J Gastroenterol 2011, 46(Suppl 1):63-69.

16. Scatarige JC, Scott WW, Donovan PJ, Siegelman SS, Sanders RC: Fatty infiltration of the liver: ultrasonographic and computed tomographic correlation. J Ultrasound Med 1984, 3(1):9-14.
17. Saadeh S, Younossi ZM, Remer EM, Gramlich T, Ong JP, Hurley M, Mullen KD, Cooper JN, Sheridan MJ: The utility of radiological imaging in nonalcoholic fatty liver disease. Gastroenterology 2002, 123(3):745-750.

18. Quinn SF, Gosink BB: Characteristic sonographic signs of hepatic fatty infiltration. AJR Am J Roentgenol 1985, 145(4):753-755.

19. Chobanian AV, Bakris GL, Black HR, Cushman WC, Green LA, Izzo JJ, Jones DW, Materson BJ, Oparil S, Wright JJ, et al: The Seventh Report of the Joint National Committee on Prevention, Detection, Evaluation, and Treatment of High Blood Pressure: the JNC 7 report. JAMA 2003 289(19):2560-2572.

20. Expert Committee on the Diagnosis and Classification of Diabetes Mellitus: Report of the expert committee on the diagnosis and classification of diabetes mellitus. Diabetes Care 2003, 26(Suppl 1):S5-S20.

21. Expert Panel on Detection, Evaluation, and Treatment of High Blood Cholesterol in Adults: Executive Summary of The Third Report of The National Cholesterol Education Program (NCEP) Expert Panel on Detection, Evaluation, And Treatment of High Blood Cholesterol In Adults (Adult Treatment Panel III). JAMA 2001, 285(19):2486-2497.

22. Tan CE, Ma S, Wai D, Chew SK, Tai ES: Can we apply the National Cholesterol Education Program Adult Treatment Panel definition of the metabolic syndrome to Asians? Diabetes Care 2004, 27(5):1182-1186.

23. Farrell GC, Chitturi S, Lau GK, Sollano JD: Guidelines for the assessment and management of non-alcoholic fatty liver disease in the Asia-Pacific region: executive summary. J Gastroenterol Hepatol 2007, 22(6):775-777.

24. Fan JG, Jia JD, Li YM, Wang BY, Lu LG, Shi JP, Chan LY: Guidelines for the diagnosis and management of nonalcoholic fatty liver disease: update 2010 (published in Chinese on Chinese Journal of Hepatology 2010; 18:163-166). J Dig Dis 2011, 12(1):38-44.

25. Vernon G, Baranova A, Younossi ZM: Systematic review: the epidemiology and natural history of non-alcoholic fatty liver disease and non-alcoholic steatohepatitis in adults. Aliment Pharmacol Ther 2011, 34(3):274-285.

26. Fan JG, Farrell GC: Epidemiology of non-alcoholic fatty liver disease in China. J Hepatol 2009, 50(1):204-210.

27. Li H, Wang YJ, Tan K, Zeng L, Liu L, Liu FJ, Zhou TY, Chen EQ, Tang H: Prevalence and risk factors of fatty liver disease in Chengdu, Southwest China. Hepatobiliary Pancreat Dis Int 2009, 8(4):377-382.

28. Fan JG, Zhu J, Li XJ, Chen L, Li L, Dai F, Li F, Chen SY: Prevalence of and risk factors for fatty liver in a general population of Shanghai, China. J Hepatol 2005, 43(3):508-514

29. Zhou YJ, Li YY, Nie YQ, Ma JX, Lu LG, Shi SL, Chen MH, Hu PJ: Prevalence of fatty liver disease and its risk factors in the population of South China. World J Gastroenterol 2007, 13(47):6419-6424.

30. Lee K, Sung JA, Kim JS, Park TJ: The roles of obesity and gender on the relationship between metabolic risk factors and non-alcoholic fatty liver disease in Koreans. Diabetes Metab Res Rev 2009, 25(2):150-155.

31. Ruhl CE, Everhart JE: Determinants of the association of overweight with elevated serum alanine aminotransferase activity in the United States. Gastroenterology 2003, 124(1):71-79.

32. Omagari K, Kadokawa Y, Masuda J, Egawa I, Sawa T, Hazama H, Ohba K, Isomoto H, Mizuta Y, Hayashida K, et al: Fatty liver in non-alcoholic non-overweight Japanese adults: incidence and clinical characteristics. J Gastroenterol Hepatol 2002, 17(10):1098-1105

33. Singh SP, Nayak S, Swain M, Rout N, Mallik RN, Agrawal O, Meher C, Rao M: Prevalence of nonalcoholic fatty liver disease in coastal eastern India: a preliminary ultrasonographic survey. Trop Gastroenterol 2004, 25(2):76-79.

34. Romeo S, Kozlitina J, Xing C, Pertsemlidis A, Cox D, Pennacchio LA, Boerwinkle E, Cohen JC, Hobbs HH: Genetic variation in PNPLA3 confers susceptibility to nonalcoholic fatty liver disease. Nat Genet 2008, 40(12):1461-1465.

35. Daly AK, Ballestri S, Carulli L, Loria P, Day CP: Genetic determinants of susceptibility and severity in nonalcoholic fatty liver disease. Expert Rev Gastroenterol Hepatol 2011, 5(2):253-263.

36. Anstee QM, Daly AK, Day CP: Genetics of alcoholic and nonalcoholic fatty liver disease. Semin Liver Dis 2011, 31(2):128-146.

37. Angulo P: Gl epidemiology: nonalcoholic fatty liver disease. Aliment Pharmacol Ther 2007, 25(8):883-889.

38. Ryu S, Chang Y, Kim SG, Cho J, Guallar E: Serum uric acid levels predict incident nonalcoholic fatty liver disease in healthy Korean men. Metabolism 2011, 60(6):860-866.

39. Xu C, Yu C, Xu L, Miao M, Li Y: High serum uric acid increases the risk for nonalcoholic Fatty liver disease: a prospective observational study. PLOS One 2010, 5(7):e11578. 
40. Lee JW, Cho YK, Ryan M, Kim H, Lee SW, Chang E, Joo KJ, Kim JT, Kim BS, Sung KC: Serum uric Acid as a predictor for the development of nonalcoholic Fatty liver disease in apparently healthy subjects: a 5-year retrospective cohort study. Gut Liver 2010, 4(3):378-383.

41. Li Y, Xu C, Y U C, Xu L, Miao M: Association of serum uric acid level with non-alcoholic fatty liver disease: a cross-sectional study. J Hepatol 2009, 50(5):1029-1034

42. Bedogni G, Miglioli L, Masutti F, Tiribelli C, Marchesini G, Bellentani S: Prevalence of and risk factors for nonalcoholic fatty liver disease: the Dionysos nutrition and liver study. Hepatology 2005, 42(1):44-52.

43. Angulo P: Obesity and nonalcoholic fatty liver disease. Nutr Rev 2007, 65(6 Pt 2):S57-S63.

doi:10.1186/1471-230X-12-123

Cite this article as: Hu et al:: Prevalence and factors associated with nonalcoholic fatty liver disease in shanghai work-units. BMC

Gastroenterology 2012 12:123.

\section{Submit your next manuscript to BioMed Central and take full advantage of:}

- Convenient online submission

- Thorough peer review

- No space constraints or color figure charges

- Immediate publication on acceptance

- Inclusion in PubMed, CAS, Scopus and Google Scholar

- Research which is freely available for redistribution 